\title{
Parallel Charge-Transfer Mechanisms Allow for Diversifying the Choices for Photoredox Catalysts
}

\author{
Katarzyna Goliszewska, ${ }^{\dagger}$ Katarzyna Rybicka-Jasińska, ${ }^{\dagger}$ John A. Clark, ${ }^{\dagger}$ Valentine I.

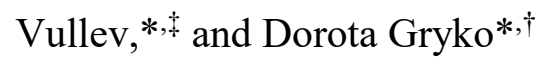 \\ †Institute of Organic Chemistry Polish Academy of Sciences, Kasprzaka 44/52, 01-224 \\ Warsaw, Poland \\ ${ }^{\ddagger}$ Department of Bioengineering, University of California, Riverside, California 92521, US
}

\begin{abstract}
Photochemistry provides access to reactive intermediates that are often inaccessible by any other means. Most organic molecules, however, are colorless ultraviolet absorbers. Therefore, photocatalysts absorbing in the visible spectral region are essential for transferring the required energy and charges to make challenging chemical transformations possible. A selection of a photocatalyst for driving oxidative or reductive reactions is crucial and is commonly based on their electrochemical potentials as well as the potentials of the starting materials. This selection, however, sometimes proves limiting and misleading, especially when the thermodynamic driving forces of the charge-transfer steps are relatively small. Here, we show that porphyrinoids with differences in their electrochemical potentials exceeding $0.5 \mathrm{~V}$ can photocatalyze the same model reaction of $N$-alkyl-2,4,6triphenylpyridinium salt with alkynyl $p$-tolylsulfone to form the same alkylated alkynyl product in similar yields. Our studies reveal that switching between parallel reaction pathways makes the attainment of these conversion efficiencies possible. Electron-rich catalysts drive the formation of alkyl radicals principally via a photoinduced electron transfer to the pyridinium ion and a sequential hole transfer recovers their ground states, i.e., PET-HT mechanism. Conversely, a photoinduced hole transfer dominates the initial formation of the reduced forms of electron-deficient porphyrins that then transfer electrons to the pyridinium salt to release the same alkyl radicals, i.e., PHT-ET mechanism. This discovery demonstrates a paradigm where reaction mechanism adjust to the electronic properties of catalysts and opens doors for transformative diversification and broadening of the applicability of photochemical transformations.
\end{abstract}




\section{INTRODUCTION}

Over the past decade, the renaissance of photochemistry has made it one of the most dynamically developing branches of organic chemistry. Activation of organic molecules, mediated by visible light, enables a large number of organic transformations including oxidations, ${ }^{1-3}$ reductions, ${ }^{4}$ formation of carbon-carbon ${ }^{5-7}$ and carbon-heteroatom ${ }^{8,9}$ bonds, and functionalization of unactivated $\mathrm{C}-\mathrm{H}$ bonds. ${ }^{10,11}$ It is possible to sustain the pace of breakthroughs in this field, however, only if deepening the mechanistic understanding of light driven reactions accompanies the development of new synthetic methods. Therefore, numerous recent reports extensively highlight the importance of filling the growing gap between utilitarian experimental results and fundamental mechanistic investigations. ${ }^{12-14}$

While in the early years of photoredox chemistry serendipity and empirical deductions drove the choices for photocatalysts (PCs), nowadays the relationships between the electrochemical and optical properties of the PCs and the substrates defines the principal basis for the PC selection. Structure-property relationships for PCs based on transition metal complexes represent a principal focus of the field. ${ }^{15-17}$ Conversely, the effects of structural modifications of organic dyes on their propensity to catalyze photochemical transformations still remains largely unexplored. Indeed, introduction of halogens, electron-withdrawing or electrondonating substituents into the structures of dyes significantly changes their redox properties, excited-state lifetimes, photostability and fluorescence quantum yields. ${ }^{18-21}$ While previous reports give guidelines how to control the oxidizing and reducing propensities of PCs, to the best of our knowledge, the means for controlling photochemical reaction mechanisms remains unknown. This gap in the knowledge places a high demand for comprehensive research endeavors focusing on the factors affecting photoredox reaction pathways and their relationship with the structural features of the PCs.

In the first instance, it is essential to clarify the use of the term "photocatalysis." It can refer to exergonic reactions where the light excitation aids to overcome large activation-energy barriers, i.e., "classic" catalysis. ${ }^{22}$ It also describes endergonic reactions where light drives the transformation to products that have higher energy than the starting materials. Viewing light as a high-energy "reagent" that contributes to the thermodynamic balance of the photo-initiated reactions allows photocatalysis to drive a considerably broader set of reactions than "classic" catalysis can, and break out of the limits of microscopic reversibility of thermal pathways. 
For extensive investigations of the relationships between the PC electronic properties, photocatalytic activity and possible modes of operation, a selection of PCs model that are relatively easy to modify is essential. Following our previous work, ${ }^{23,24}$ we employ porphyrinoids as PCs. Porphyrins are well-known photocatalyst not only for singlet oxygen generation (photosensitizers), ${ }^{25}$ but also for single-electron oxidation and reduction of organic molecules (photoredox catalysts). ${ }^{23,24}$ The facility of introducing electron-withdrawing or electron-donating substituents and metal ion, or of modifying the whole macrocyclic ring makes porphyrinoids particularly desirable PCs for systematic mechanistic studies. In addition, enriching the knowledge about photoredox activity of porphyrins is crucial for broadening their use in visible-light mediated reactions.

Herein, we report mechanistic studies on porphyrins as photocatalysts where varying the substituents and the state of metalation allows for adjusting their electrochemical potentials over ranges exceeding half a volt. These drastic changes in the redox properties of the porphyrins did not alter by much the overall yields of the catalyzed reaction that involves reduction of a pyridinium salt as a key step. The analysis reveals an interplay between two parallel mechanisms that allows electron-rich and electron-deficient photocatalysts to drive the same reaction to completion. That is, the reaction mechanism and pathways adjust to the electronic properties of the catalysts.

\section{RESULTS AND DISCUSSION}

Model reaction. Recently we reported a visible-light induced deaminative alkynylation with Katritzky salts using eosin Y (EY) as photocatalyst (Scheme 1). ${ }^{26}$

Scheme 1. Model deaminative alkynylation with Katritzky salts.

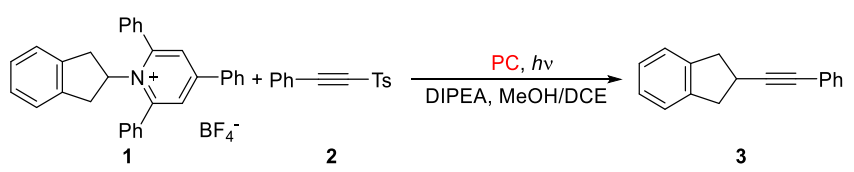

The reaction involves reductive activation of the pyridinium cation of salt $\mathbf{1}$, where light drives electrons to it from a sacrificial donor, e.g., a tertiary amine, such as DIPEA, via a sequence of photosensitizer-mediated charge-transfer (CT) steps (Scheme 2). The photochemically formed dihydropyridine radical A releases alkyl radical $\mathbf{B}$ needed for the alkynylation. An addition of radical $\mathbf{B}$ to alkyne $\mathbf{2}$, followed by elimination of the tosyl radical yielding product $\mathbf{3}$, is a key step in this transformation. ${ }^{26}$ Converting the amine into pyridinium salt allows for a facile 
cleavage of the C-N bond and the electron-deficient reactant is an activated derivative of the terminal alkyne.

Scheme 2. Plausible mechanistic pathways.

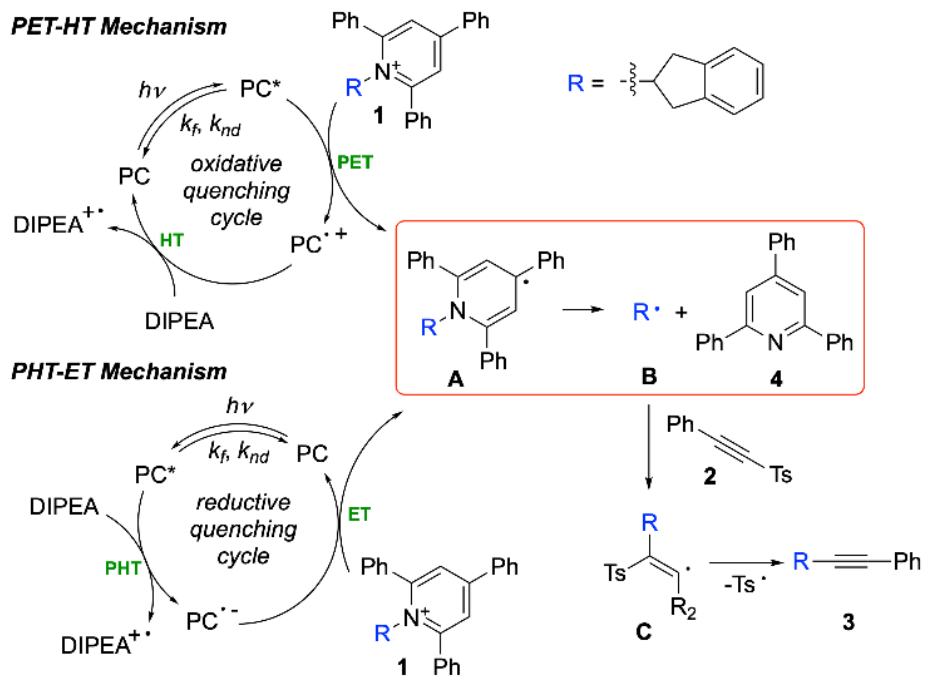

Stern-Volmer analysis reveals that pyridinium salt $\mathbf{1}$ quenches the emission of EY more efficiently than DIPEA $\left(k_{q(\mathbf{1})}=3.8 \times 10^{10} \mathrm{~s}^{-1} \mathrm{M}^{-1}\right.$ vs. $\left.k_{q(\text { DIPEA })}=2.3 \times 10^{9} \mathrm{~s}^{-1} \mathrm{M}^{-1}\right){ }^{26}$ This order-ofmagnitude difference between the quenching rate constants indicates that the prevalent pathway of the reaction involves photoinduced electron transfer (PET) from $\mathbf{P C}^{*}$ to the pyridinium ion of salt 1 and subsequent reduction of PC $^{{ }^{++}}$by a hole transfer (HT) to DIPEA, i.e., PET-HT Mechanism. While electron transfer (ET) is charge transfer (CT) that involves a transfer of an electron to a lowest unoccupied molecular orbital (LUMO), HT is CT involving a transfer of an electron from a highest occupied molecular orbital (HOMO) to a singly occupied orbital, which is equivaled to moving a vacancy from a singly occupied orbital (i.e., the hole) to a higher lying HOMO. ${ }^{27}$ The quenching by salt $\mathbf{1}$ appears to exceed the bimolecular diffusion-limited rates, ${ }^{28}$ suggesting for static mechanism involving ground-state aggregation of the positively charged pyridinium cation with the negatively charged EY. It is consistent with favorable electrostatic interactions despite the steric hindrance imposed by the three phenyl substituents on the cation of salt 1. Furthermore, PET leads to electroneutral pyridinium radical A, eliminating the electrostatic attraction that allows the aggregate to dissociate after this first $\mathbf{C T}$ step, which decreases the likelihood for undesired back CT. The fact that DIPEA also quenches the PC suggests for a possibly minor contribution from a parallel pathway that involves photoinduced hole transfer (PHT) from $\mathbf{P C}{ }^{*}$ to the tertiary amine and consequent reduction of pyridinium salt 1 by the thus generated PC•-, i.e., PHT-ET Mechanism (Scheme 2). Can this 
second mechanism have any consequential importance? (Meanwhile, we should emphasize that the generic use of PC does not represent its charge. Because $\mathbf{E Y}$ is doubly negatively charged at $\mathrm{pH}$ above $4, \mathrm{PC}=\mathrm{EY}^{2-},{ }^{29} \mathrm{PC}^{{ }^{+}}=\mathrm{EY}^{\bullet^{-}}$and $\left.\mathrm{PC}^{\cdot-}=\mathrm{EY}^{\cdot 3-}\right)$.

The generated dihydropyridine radical $\mathbf{A}$ is pronouncedly stable. For chlorinated solvents, such as DCM, the electrochemical reduction of pyridinium shows practically complete chemical reversibility at moderate scan rates (Figure 1). Conversely, $\mathrm{MeOH}$ induces a loss in the reversible behavior, which is consistent with fragmentation of dihydropyridine radical $\mathbf{A}$ to alkyl radical $\mathbf{B}$ and pyridine 4 occurring in timescales of se-conds (Figure 1d). Furthermore, addition of alkyne $\mathbf{2}$ does not alter the voltammograms of salt $\mathbf{1}$ (Figure 1a,b), which allows for ruling out possibilities for direct interactions between salt $\mathbf{1}$ and alkyne $\mathbf{2}$, most likely because of the steric hindrance from the three phenyl substituents of salt $1 .{ }^{30}$ Concurrently, radicaltrapping studies confirm the formation of $\mathbf{B}$ (see SI).

a)

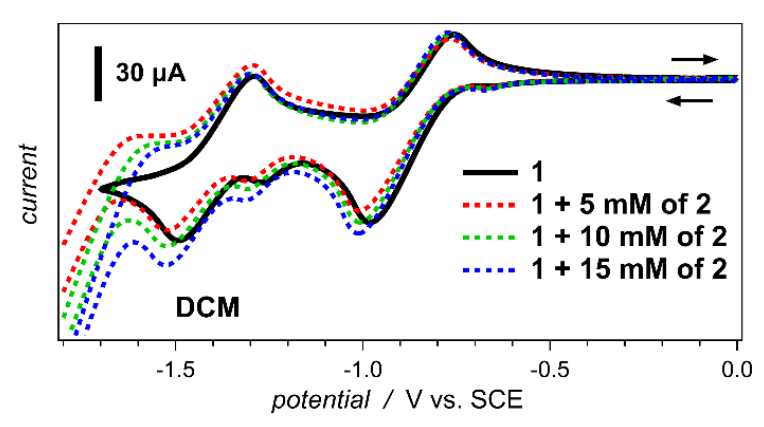

c)

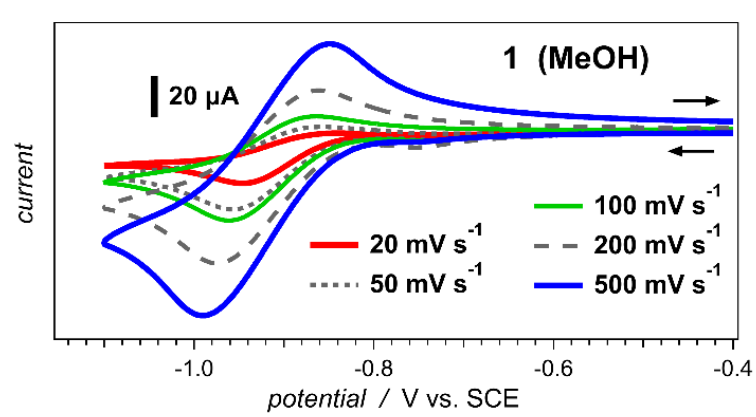

b)

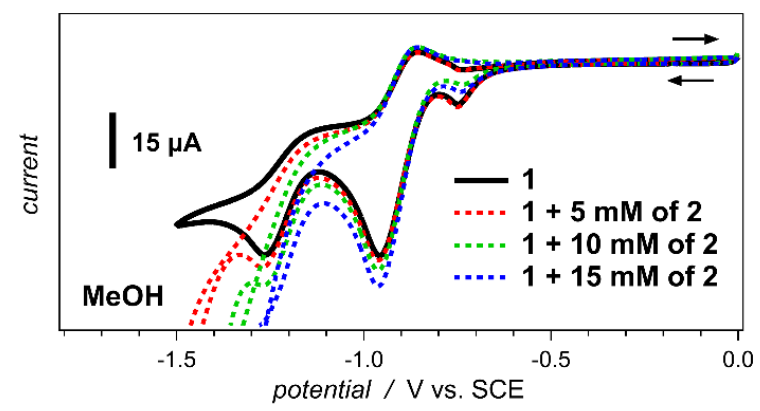

d)

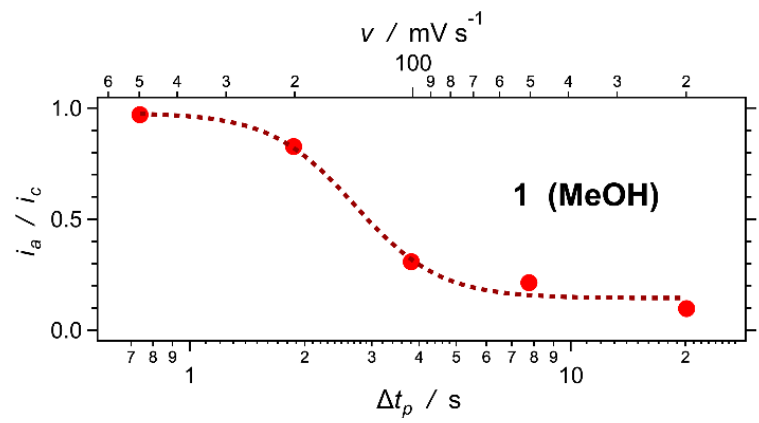

Figure 1. Electrochemical behavior of pyridinium salt 1. (a,b) Cyclic voltammograms of 1 in the presence of various amounts of the alkyne 2 for DCM and $\mathrm{MeOH}$ recorded at a scan rate, $v=100 \mathrm{mV} \mathrm{s}^{-1}$. (c) Cyclic voltammograms of 1 for $\mathrm{MeOH}$ at different scan rates. (d) Dependence of the ratio between the anodic, $i_{a}$, and the cathodic, $i_{c}$, peaks (from c) on the scan rate and on the time delay between the two peaks, $\Delta t_{p}$, showing loss of reversibility when $\Delta t_{p}$, exceeds about $2 \mathrm{~s}$. All voltammograms are recorded in the presence of $100 \mathrm{mM} \mathrm{N}(n$ $\mathrm{Bu})_{4} \mathrm{ClO}_{4}$.

These results concur with a sequential formation of intermediates $\mathbf{B}, \mathbf{C}$, and product $\mathbf{3}$. While the radical release, leading to product $\mathbf{3}$, occurs in seconds, the rates of the photoinduced $\mathbf{C T}$ 
steps are in the nanosecond and sub-nanosecond time domains. That is, the rates of PET and PHT have to be comparable or larger than the rates of radiative and non-radiative deactivation of ${ }^{1} \mathbf{P C} *$. Therefore, considering the thermodynamics of the different $\mathbf{C T}$ steps, i.e., ensuring that $\Delta G^{(0)}<0$, suffices as guidelines for selecting the PCs for this reaction.

Photocatalysts. In the model reaction, the photocatalyst drives the endergonic electron transfer from the sacrificial donor to the pyridinium ion, i.e., for DCM in the presence of $0.1 \mathrm{M}$ electrolyte, $E_{\mathrm{DIPEA}^{*+} / \mathrm{DIPEA}^{(0)}}^{(0)}=0.86 \mathrm{~V}$ vs. SCE and $E_{\mathrm{Py}^{+} / \mathrm{Py}^{\circ}}^{(0)}=-0.83 \mathrm{~V}$ vs. SCE. The optical excitation energy (or zero-to-zero energy, $E_{00}$ ) of the photocatalyst should overcome the hugely unfavorable driving force of this CT process: $E_{00}>\Delta G_{\mathrm{CT}^{(0)}}^{(0)} F\left(E_{\mathrm{DIPEA}^{-+} / \mathrm{DIPEA}}^{(0)}-E_{\mathrm{Py}^{+} / \mathrm{Py}^{*}}^{(0)}\right)=$ $1.69 \mathrm{eV}$ where $F$ is the Faraday constant. The "uphill" $\Delta G_{\mathbf{C T}}{ }^{(0)} \leq 1.69 \mathrm{eV}$ equates to the energy of 735-nm near infrared light radiation. Therefore, the bathochromic edge of the absorption spectrum of the photocatalyst should be in the visible spectral region, and many dyes can meet this requirement. Furthermore, the reduction potentials of a PC for this reaction should bracket those of the sacrificial donor and the pyridinium acceptor, i.e., $E_{\mathrm{PC} / \mathrm{PC}^{\cdot-}}^{(0)}<E_{\mathrm{Py}^{+} / \mathrm{Py}^{*}}^{(0)}$ and $E_{\mathrm{PC}^{*+} / \mathrm{PC}}^{(0)}>E_{\mathrm{DIPEA}^{*+} / \mathrm{DIPEA}^{*}}^{(0)}$

Similar to EY, which we previously used, ${ }^{26}$ the electrochemical and optical properties of tetraphenylporphyrin (9, Figure 2) appear to meet the requirements for photocatalyzing the
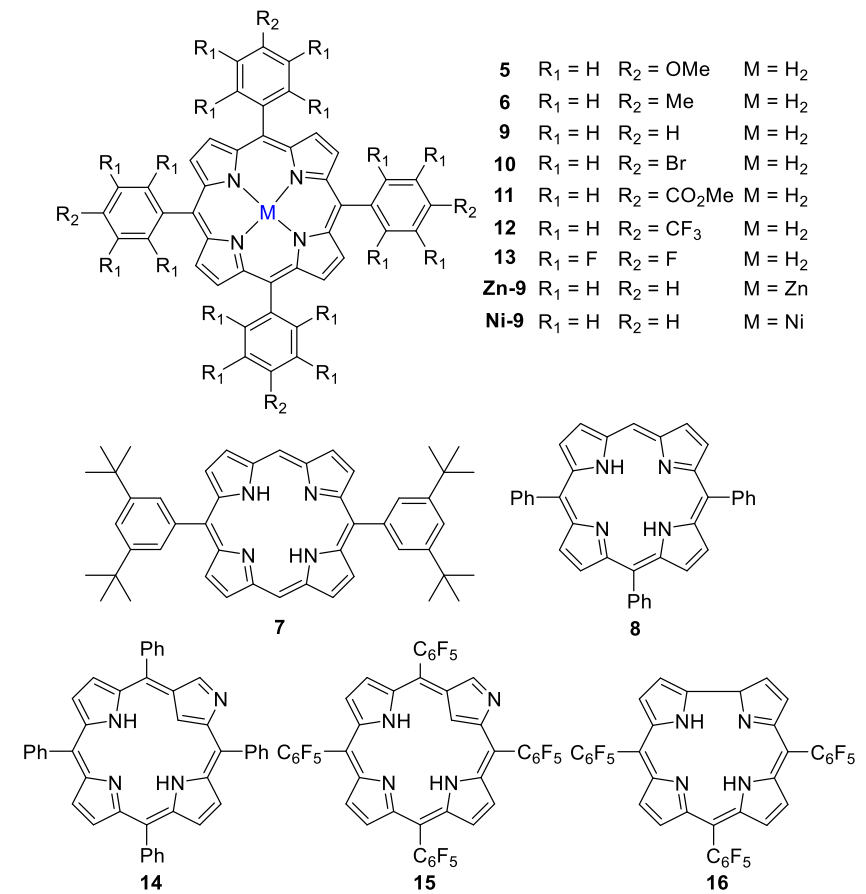

Figure 2. Porphyrinoids selected as photocatalysts for mechanistic studies. 
model reaction. Unlike EY, however, the structure of porphyrin 9 provides a myriad of possibility for attaining derivatives with a wide range of electronic properties via, for example, metalation or variation of the substituents on the phenyls, i.e., $\mathrm{R}_{1}$ and $\mathrm{R}_{2}$. In contrast, $\mathbf{E Y}$ has only the four sites on the xanthene rings, occupied by the bromines, along with the four sites on the phenyl, that are prone to modifications, which can potentially affect its electronic properties. ${ }^{31}$ For mechanistic studies, therefore, we select a series of porphyrinoids 5-16 taking under consideration their (1) commercial availability or straightforwardness of their synthesis, (2) solubility, and above all, (3) diversity of their structural features and redox properties.

While changing the substituents, along with incorporating different metal ions, does not truly alter the optical excitation energy $E_{00}$, it drastically varies the reduction potentials of oxidation between about 0.8 and $1.6 \mathrm{~V}$ vs. SCE, and of reduction - between -1.3 and $-0.7 \mathrm{~V}$ vs. SCE (Table 1). As expected, porphyrins with electron-withdrawing substituents exhibit low reducing propensities (e.g., 12 and 13), while the electron-rich derivatives can act as strong reductants (e.g., $\mathbf{5}$ and Zn-9) (Table 1).

Table 1. Electrochemical potentials and zero-to-zero excitation energy of the tested photocatalysts.

\begin{tabular}{cccccc}
\hline Entry & $\mathbf{P C}$ & $\boldsymbol{E}_{\mathbf{P C} / \mathbf{P C}^{--}}^{(\mathbf{( 0 )}}\left[\mathrm{V}^{\mathrm{a}}\right.$ & $\boldsymbol{E}_{\mathbf{P C}^{++} / \mathbf{P C}}^{(\mathbf{0})}[\mathrm{V}]^{\mathrm{a}}$ & $\boldsymbol{E}_{\mathbf{E C H}}[\mathrm{eV}]^{\mathrm{b}}$ & $\boldsymbol{E}_{\mathbf{0 0}}[\mathrm{eV}]^{\mathbf{c}}$ \\
\hline 1 & $\mathbf{5}$ & -1.24 & 0.91 & 2.15 & 1.90 \\
2 & $\mathbf{6}$ & -1.21 & 1.00 & 2.21 & 1.90 \\
3 & $\mathbf{7}$ & -1.17 & 1.06 & 2.23 & 1.96 \\
4 & $\mathbf{8}$ & -1.17 & 1.11 & 2.28 & 1.94 \\
5 & $\mathbf{9}$ & -1.20 & 1.04 & 2.24 & 1.91 \\
6 & $\mathbf{1 0}$ & -1.10 & 1.15 & 2.25 & 1.91 \\
7 & $\mathbf{1 1}$ & -1.05 & 1.21 & 2.26 & 1.91 \\
8 & $\mathbf{1 2}$ & -1.06 & 1.23 & 2.29 & 1.92 \\
9 & $\mathbf{1 3}$ & -0.74 & 1.63 & 2.37 & 1.94 \\
10 & $\mathbf{Z n - 9}$ & -1.34 & 0.78 & 2.12 & 2.10 \\
11 & $\mathbf{N i}-\mathbf{9}$ & -1.24 & 1.11 & 2.35 & 1.90 \\
12 & $\mathbf{1 4}$ & $-0.93^{\mathrm{d}}$ & $0.96^{\mathrm{d}}$ & 1.89 & 1.71 \\
13 & $\mathbf{1 5}$ & $-1.19^{\mathrm{d}}$ & $1.06^{\mathrm{d}}$ & 2.25 & 1.73 \\
14 & $\mathbf{1 6}$ & $-1.00^{\mathrm{d}}$ & $1.10^{\mathrm{d}}$ & 2.10 & 2.00 \\
15 & $\mathbf{E Y}$ & -1.08 & 0.76 & 1.84 & 2.31 \\
\hline
\end{tabular}

${ }^{\text {a}}$ From cyclic voltammetry measurements for DCM in the presence of $100 \mathrm{mM}\left(n-\mathrm{C}_{4} \mathrm{H}_{9}\right)_{4} \mathrm{NClO}_{4}$, vs. SCE, ${ }^{\mathrm{b}}$ Electrochemical HOMO-LUMO gap, $E_{\mathrm{ECH}} \approx F\left(E_{\mathrm{PC}{ }^{*+} / \mathrm{PC}}^{(0)}-E_{\mathrm{PC} / \mathrm{PC}{ }^{-}-}^{(0)}\right.$, where $F$ is the Faraday constant, ${ }^{\mathrm{c}}$ From steady-state optical absorption and emission spectra for DCM, ${ }^{\mathrm{d}}$ From the inflection point of voltammogramic waves showing irreversible behavior. ${ }^{32}$ 
For porphyrinoids the electrochemical HOMO-LUMO gaps, $E_{\mathrm{ECH}}$, as estimated from the differences between the reduction potentials, are up to $400 \mathrm{meV}$ larger than the optical HOMOLUMO gaps, as represented by $E_{00}$ obtained from steady-state optical absorption and emission spectra (Table 1, Figure 3). It suggests for considerable differences between the energies of the solvated ions and the photoexcited species as a corollary of favorable interactions between electrons and holes on spatially-overlapping singly-occupied molecular orbitals in ${ }^{\mathbf{1}} \mathbf{P C} *{ }^{33}$ These relatively large differences between $E_{00}$ and $E_{\mathrm{ECH}}$ place further constrains on the plausibility of the photoinduced CT steps. Specifically, the excited-state photocatalysts may not be a good enough electron donors to reduce the pyridinium ion or a good enough electron acceptors to oxidize DIPEA. That is, the electronic properties of the selected chromophores shoud meet the PC requirements for the model reaction. To survey the propensity of the selected PCs to photocatalyze the model reaction of 2,4,6-triphenylpyridinium cation (introduced as salt 1) with alkynyl $p$-tolylsulfone 2 we employ the reaction conditions that we previously established for $\mathbf{E Y} .{ }^{26}$ As evident from the loss of the anodic wave (Figure 1c,d), methanol solvent medium is essential for producing the alkyl radical $\mathbf{B}$ (Scheme 2). To ensure complete solubilization of the porphyrinoids in the reaction solution, we lower the catalyst loading to 0.1 mol\%. Despite the low PC loading, the reaction still affords desired product $\mathbf{3}$ in quite decent yields, reflecting the robustness of these catalysts for deaminative alkynylation.
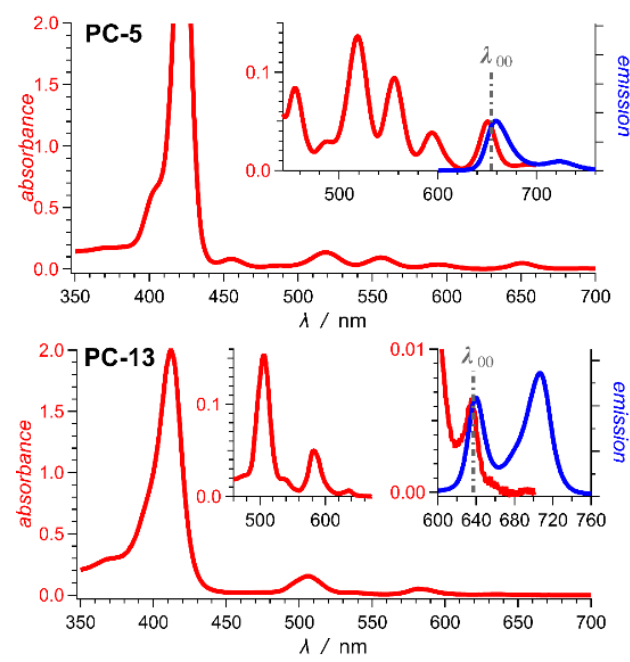

Figure 3. The steady-state optical absorption and emission spectra of PC $5\left(2.2 \times 10^{-6} \mathrm{M}\right)$ and $13\left(2.6 \times 10^{-6} \mathrm{M}\right)$, showing the designation of the wavelength, $\lambda_{00}$, used for estimating $E_{00}\left(\lambda_{\mathrm{ex}}=422\right.$ and $412 \mathrm{~nm}$ for $\mathbf{5}$ and $\mathbf{1 3}$ respectively).

All tested porphyrins (5 to 13, along with $\mathbf{Z n - 9}$ and Ni-9) exhibit good catalytic activity (Table 2). 
Table 2. Deaminative alkynylation catalyzed by porphyrinoids. ${ }^{a}$

\begin{tabular}{ccc}
\hline Entry & PC & Yield [\%] $^{b}$ \\
\hline 1 & $\mathbf{5}$ & 59 \\
2 & $\mathbf{6}$ & 50 \\
3 & $\mathbf{7}$ & 54 \\
4 & $\mathbf{8}$ & 54 \\
5 & $\mathbf{9}$ & 55 \\
6 & $\mathbf{1 0}$ & 49 \\
7 & $\mathbf{1 1}$ & 53 \\
8 & $\mathbf{1 2}$ & 64 \\
9 & $\mathbf{1 3}$ & 66 \\
10 & $\mathbf{Z n - 9}$ & 68 \\
11 & $\mathbf{N i - 9}$ & 56 \\
12 & $\mathbf{1 4}$ & 8 \\
13 & $\mathbf{1 5}$ & 9 \\
14 & $\mathbf{1 6}$ & 9 \\
\hline
\end{tabular}

${ }^{a}$ Reaction conditions: alkyne $2(0.10 \mathrm{mmol})$, pyridinium salt 1 (1.4 equiv.), PC (0.1 mol\%), DIPEA (3.25 equiv.), $\mathrm{MeOH} / \mathrm{DCE}(\mathrm{c}=0.033 \mathrm{M}), 2: 1$ volume ratio that corresponds to molar ratio, $\chi_{\mathrm{DCE}}=0.2$, ambient temperature $(20$ $22{ }^{\circ} \mathrm{C}$ ), $16 \mathrm{~h}$, under Ar atmosphere, light source: green LED diode (for more details see SI), ${ }^{b} \mathrm{GC}$ yield.

Conversely, the $N$-confused porphyrins 14 and 15 and corrole 16, do not prove truly efficient in catalyzing the model reaction (entries 12-14), which we attribute to the lack of chemical reversibility of their electrochemical reduction and oxidation (see SI). ${ }^{32,34}$ After the initial photoinduced charge transfer (PCT), therefore side reactions, such as degradation of the radical ions of 14, 15, and 16, compete with the second CT steps essential for the PC recovery, resulting in depletion of the PCs in a certain number of cycles.

This finding points to another important requirement for a photoredox catalyst: it has to exhibit reversible electrochemical oxidation and reduction behavior. Irreversibility of the voltammograms does not necessarily render the PC unfeasible. The timescales of the electrochemical measurements employing slow to moderate scan rates tend to be orders of magnitude longer that the time constants of the CT steps, especially at high substrate loading. Reversible electrochemical behavior, however, ensures the stability of the oxidized and reduced forms of the PC, which is essential for enduring a large number of turnovers. ${ }^{32,34}$

Mechanistic considerations. The excited-state lifetimes $(\tau)$ of almost all porphyrins range between about 8 and $11 \mathrm{~ns}$ (see SI), which appear to be long enough for the PCT steps that drive the chemical transformations. Even reactions catalyzed by porphyrins $\mathbf{1 0}$ and Zn-9 with relatively short lifetimes (i.e., $\tau_{\mathbf{1 0}}=1.48 \mathrm{~ns}$ and $\tau_{\mathrm{Zn}-\mathbf{9}}=2.29 \mathrm{~ns}$, see SI) give yields that are quite 
comparable to those from the other PCs. Furthermore, all PCs exhibit emission quantum yields that show no correlation with the obtained reaction yields. These findings strongly suggest that the quantum yields of the PCT steps are not the limiting factor for obtaining the large overall reaction yields that we observe. Conversely, the substituent-induced broad variation of the electrochemical properties of the porphyrins poses a conundrum that reveals underlying complexity of the mechanistic scenarios and an interplay of alternative pathways. An increase in the electron-withdrawing strength of the substituents, for example, should make a PC electron-deficient enough to completely shut down the initial PET with the pyridinium ion of salt 1 and make the PET-HT Mechanism impossible. Nevertheless, electron-deficient tetra(pentafluorophenyl)porphyrin (13) is as effective $\mathbf{P C}$ as the electron-rich porphyrin $\mathbf{5}$. The insufficiently negative reduction potentials of catalyst 13 (Table 1, Figure 4), indicate that it is not a good enough electron donor to photoreduce 2,4,6-triphenylpyridinium cation of salt $\mathbf{1}$, which renders the PET-HT Mechanism implausible.

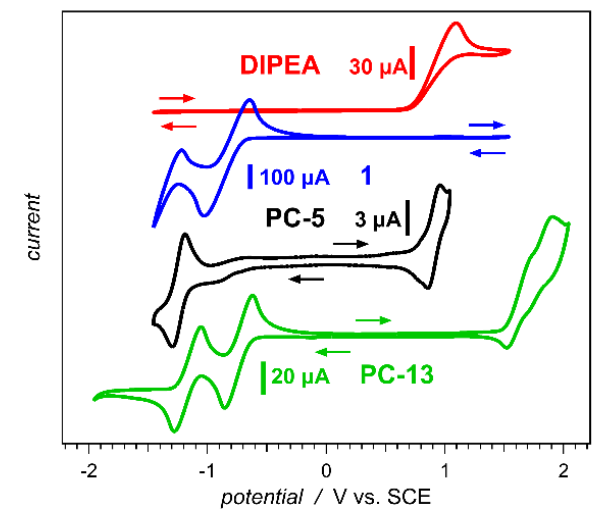

Figure 4. Cyclic voltammograms of DIPEA, 1 and the PCs 5 and $\mathbf{1 3}$ for DCM in the presence of $100 \mathrm{mM} \mathrm{N}(n$ $\mathrm{Bu})_{4} \mathrm{ClO}_{4}$ recorded at a scan rate, $v=100 \mathrm{mV} \mathrm{s}^{-1}$.

An alternative pathway, however, involving for example the PHT-ET Mechanism (Scheme 2), can lead to the observed high yields of the reaction catalyzed by porphyrin 13 .

Stern-Volmer (SV) analysis permits us to probe the propensity for the initial PCT (photocatalytic charge transfer) steps to occur. While using pyridinium salt $\mathbf{1}$ as a quencher allows for quantifying the bimolecular PET rate constants, quenching studies with DIPEA provide information about the PHT rate constants. In the presence of either only salt $\mathbf{1}$ or only DIPEA, all PCs, except 9, 12, 13, and Zn-9, exhibit instability in $\mathrm{MeOH}$ media. Therefore, we employ DCE for the SV analysis and for $\mathbf{9}, \mathbf{1 2}, \mathbf{1 3}$, and $\mathbf{Z n - 9}$, for others we also use $\mathrm{MeOH}$ (Table 3, Figure 5). Many of the porphyrin derivatives are inherently electron rich with $E_{00}<$ 
$E_{\mathrm{ECH}}$ (Table 1) and they do not manifest detectable emission quenching by DIPEA (Table 3, entries 1-4, 6), which precludes the PHT-ET pathway as the sole possibility for these PCs.

Furthermore, $k_{q(\mathrm{DIPEA})}<<k_{q(\mathbf{1})}$ in some cases, such as porphyrin 9 in $\mathrm{MeOH}$ and $\mathbf{Z n - 9}$ in DCE (entries 5 and 10), which suggests that, similar to EY, the PET-HT mechanism dominates and PHT-ET has a relatively minor contribution to the overall yields of the reaction driven by such electron-rich PCs. In contrast, electron-deficient PCs, such as 13, exhibit undetectable emission quenching with the pyridinium salt 1 (entry 9). Therefore, for such PCs the PHT-ET mechanism represents the only pathway toward the final product 3 .
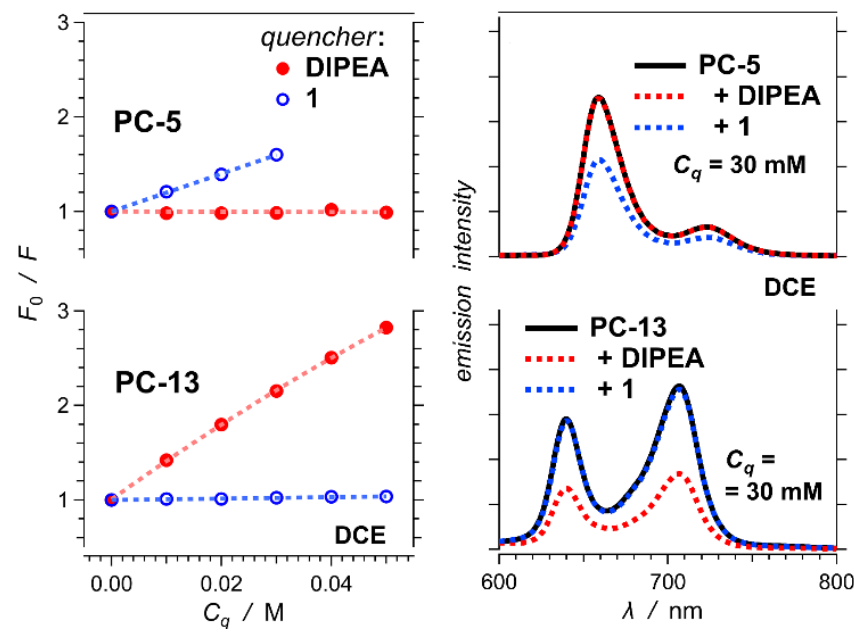

Figure 5. Stern-Volmer (SV) analysis for electron-rich and electron-deficient PCs 5 and 13, respectively, obtained from optical spectra recorded for DCE. ( $\lambda_{\mathrm{ex}}=518$ and $506 \mathrm{~nm}$ for $\mathbf{5}$ and $\mathbf{1 3}$ respectively).

Table 3. Quenching results for porphyrins measured in DCE and MeOH.

\begin{tabular}{|c|c|c|c|c|}
\hline Entry & $\mathbf{P C}$ & Solvent & $k_{q(\text { DIPEA) }}\left[\times 10^{8} \mathrm{~s}^{-1} \cdot \mathrm{M}^{-1}\right]$ & $k_{q(1)}\left[\times 10^{8} s^{-1} \cdot M^{-1}\right]$ \\
\hline 1 & 5 & DCE & $-{ }^{a}$ & $24.5 \pm 0.50$ \\
\hline 2 & 6 & DCE & $-^{\mathrm{a}}$ & $20.6 \pm 0.70$ \\
\hline 3 & 7 & DCE & $-^{\mathrm{a}}$ & $19.4 \pm 0.50$ \\
\hline 4 & 8 & DCE & $-^{\mathrm{a}}$ & $15.2 \pm 0.70$ \\
\hline \multirow[t]{2}{*}{5} & 9 & DCE & $-^{\mathrm{a}}$ & $8.06 \pm 0.71$ \\
\hline & 9 & $\mathrm{MeOH}$ & $4.68 \pm 0.31$ & $26.8 \pm 1.8$ \\
\hline 6 & 10 & DCE & $-^{\mathrm{a}}$ & $36.6 \pm 0.40$ \\
\hline 7 & 11 & DCE & $3.29 \pm 0.11$ & $4.01 \pm 0.23$ \\
\hline \multirow[t]{2}{*}{8} & 12 & DCE & $6.63 \pm 0.17$ & $8.97 \pm 0.18$ \\
\hline & 12 & $\mathrm{MeOH}$ & $9.15 \pm 0.2$ & $3.72 \pm 0.22$ \\
\hline 9 & 13 & DCE & $41.1 \pm 0.2$ & $-^{\mathrm{a}}$ \\
\hline \multirow{3}{*}{10} & 13 & $\mathrm{MeOH}$ & $50.0 \pm 0.6$ & $-{ }^{\mathrm{a}}$ \\
\hline & Zn-9 & DCE & $25.2 \pm 3.2$ & $376 \pm 11$ \\
\hline & Zn-9 & $\mathrm{MeOH}$ & $-^{\mathrm{a}}$ & $161 \pm 28$ \\
\hline 11 & Ni-9 & DCE & $-^{\mathrm{a}}$ & $23.0 \pm 1.1$ \\
\hline
\end{tabular}

${ }^{\mathrm{a}}$ Emission quenching is not detected. 
Furthermore, $k_{q(\text { DIPEA })}<<k_{q(\mathbf{1})}$ in some cases, such as porphyrin 9 in $\mathrm{MeOH}$ and $\mathbf{Z n - 9}$ in DCE (entries 5 and 10), which suggests that, similar to EY, the PET-HT mechanism dominates and PHT-ET has a relatively minor contribution to the overall yields of the reaction driven by such electron-rich PCs. In contrast, electron-deficient PCs, such as 13, exhibit undetectable emission quenching with the pyridinium salt 1 (entry 9). Therefore, for such PCs the PHT-ET mechanism represents the only pathway toward the final product 3 .

For most PCs, the estimated emission-quenching rate constants range between about $2 \times 10^{8}$ and $5 \times 10^{9} \mathrm{M}^{-1} \cdot \mathrm{s}^{-1}$, which is typical for efficient bimolecular processes. For complex $\mathbf{Z n - 9}$, however, $k_{q(1)}>1 \times 10^{10} \mathrm{M}^{-1} \cdot \mathrm{s}^{-1}$, which exceeds the diffusion limits for biomolecular reactions in condensed phase and suggests for ground-state aggregation between the zinc porphyrin and the pyridinium ion (entry 10 for DCE and $\mathrm{MeOH}$ ). Furthermore, while $k_{q(\text { DIPEA) }}=2.5 \times 10^{9} \mathrm{M}^{-}$ ${ }^{1} \cdot \mathrm{s}^{-1}$ for catalyst $\mathbf{Z n - 9}$ is smaller than the bimolecular diffusion limit, DIPEA-induced perturbation in the absorption spectra of complex $\mathbf{Z n - 9}$ suggests for ground-state aggregation between this PC and the amine (Figure 6).

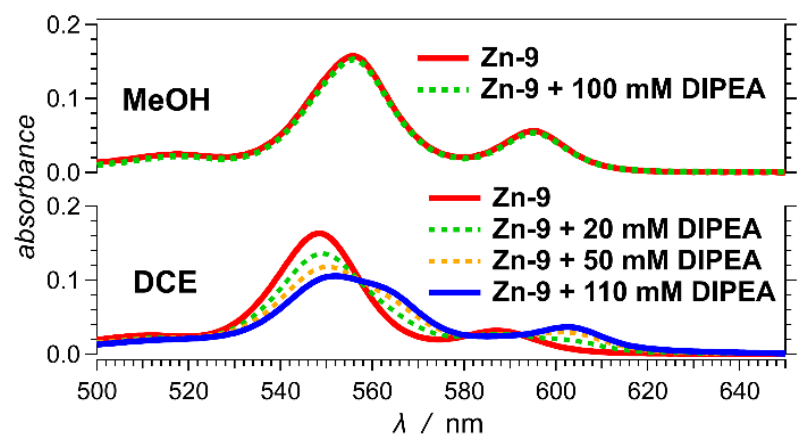

Figure 6. Solvent effects of the optical absorption spectra focused on the Q-bands of $\mathbf{Z n - 9}\left(5.8 \times 10^{-6} \mathrm{M}\right)$ in the presence of various amounts of DIPEA.

The electronic properties of the PCs correlate well with the outcomes from the SV studies that reveal the preferred pathways for the light-driven formation of radicals. As informative as the $\mathrm{SV}$ analysis is about the parallel mechanisms at play, it can examine only the plausibility and the nature of the initial photoinduced CT steps.

Charge-transfer analysis. To analyze the feasibility and the interplay between the PET-HT and PHT-ET Mechanisms, we evaluate the thermodynamic driving forces, $-\Delta G^{(0)}$, of the different CT steps involved: ${ }^{35,36}$ 
PET-HT Mechanism - oxidative quenching

PET: $\mathrm{PC}^{*}+\mathrm{Py}^{+} \rightarrow \mathrm{PC}^{\bullet}+\mathrm{Py}^{\bullet}$

$$
\begin{aligned}
& \Delta G_{\mathrm{PET}}^{(0)}=F\left(E_{\mathrm{PC} / \mathrm{PC}^{++}}^{(0)}-E_{\mathrm{Py}^{+} / \mathrm{Py}}^{(0)}\right)-E_{00}+\Delta G_{S}+W \\
& \text { HT: } \mathrm{PC}^{\cdot+}+\mathrm{DIPEA} \rightarrow \mathrm{PC}+\mathrm{DIPEA}^{\cdot+}
\end{aligned}
$$

$$
\Delta G_{\mathrm{HT}}^{(0)}=F\left(E_{\mathrm{DIPEA}^{\prime} \mathrm{DIPEA}^{\cdot+}}^{(0)}-E_{\mathrm{PC}^{*+} / \mathrm{PC}}^{(0)}\right)+\Delta G_{S}+W \quad \mathbf{1 b}
$$

PHT-ET Mechanism - reductive quenching

PHT: PC* $*$ DIPEA $\rightarrow \mathrm{PC}^{\bullet-}+$ DIPEA $^{\bullet+}$

$\Delta G_{\mathrm{PHT}}^{(0)}=F\left(E_{\mathrm{DIPEA}^{\cdot+} / \mathrm{DIPEA}^{(0)}}^{(0)} E_{\mathrm{PC} / \mathrm{PC}^{--}}^{(0)}\right)-E_{00}+\Delta G_{S}+W \mathbf{1 c}$

ET: $\mathrm{PC}^{\bullet^{-}+} \mathrm{Py}^{+} \rightarrow \mathrm{PC}+\mathrm{Py}^{\bullet}$

$\Delta G_{\mathrm{ET}}^{(0)}=F\left(E_{\mathrm{PC} / \mathrm{PC}^{--}}^{(0)}-E_{\mathrm{Py}^{+} / \mathrm{Py}}^{(0)}\right)+\Delta G_{S}+W \quad \mathbf{1 d}$

where $E_{\mathrm{PC}^{*+} / \mathrm{PC}}^{(0)}$ and $E_{\mathrm{DIPEA}^{*+} / \mathrm{DIPEA}}^{(0)}$ are the reduction potentials of the oxidized PC and sacrificial donor, respectively, and $E_{\mathrm{PC} / \mathrm{PC}}^{(0)}$ and $E_{\mathrm{Py}^{*+} / \mathrm{Py}}^{(0)}$ are the reduction potentials of the $\mathbf{P C}$ and the pyridinium acceptor, respectively. The Faraday constant, $F$, assumes a value of 1 e for calculating the energy in $\mathrm{eV} ; E_{00}$ is estimated from the steady-state absorption and emission spectra of the porphyrins (Figure 3); $\Delta G_{\mathrm{S}}$ is the Born solvation energy ${ }^{35}$ and $W$ is Coulomb work term. Overall, $W$ accounts for the $\mathbf{C T}$-induced change in the electrostatic interaction between the donor and the acceptor, while $\Delta G_{\mathrm{S}}$ accounts for the electrostatic interaction energy of the donor and the acceptor with the solvating media. The trends in the estimated CT driving forces concur with the SV results. The estimates of $\Delta G_{\mathrm{PHT}}{ }^{(0)}$ for the electron-rich PCs, manifesting emission that is not quenched by DIPEA, range between $-0.02 \mathrm{eV}( \pm 0.12 \mathrm{eV})$ for 10 to $0.13 \mathrm{eV}$ for 5 and Ni-9 (Figure 7a). Concurrently, the $\Delta G_{\mathrm{PHT}}{ }^{(0)}$ values for the electron deficient PCs, which show emission that DIPEA can quench, range between -0.5 and $-0.07 \mathrm{eV}$ (Figure 7a). Conversely, while $\Delta G_{\mathrm{PET}}^{(0)} \approx-0.5 \mathrm{eV}$ for the electron-rich $\mathbf{Z n - 9}$, for most PCs showing emission that pyridinium salt 1 quenches, the values of $\Delta G_{\mathrm{PET}}{ }^{(0)}$ cluster about \pm 0.15 $\mathrm{eV}$ around zero (Figure $7 \mathrm{~b}$ ). At the same time, the large positive values of $\Delta G_{\mathrm{PET}}{ }^{(0)}$ for the electron deficient $\mathbf{1 3}$ is consistent with the lack of quenching of its emission by $\mathbf{1}$ (Figure 5, 7b, Table 3). Therefore, for the most electron-rich and the most electron-deficient PCs, the 
estimated driving forces of the photoinduced CT provide predictions about the plausible mechanism that concur with the results from the SV analysis. When it comes to PCs for which $\Delta G^{(0)}$ assumes small positive values, i.e., $\leq 0.15 \mathrm{eV}, \mathrm{SV}$ reveals quenching of their emission by salt 1 but not by DIPEA.
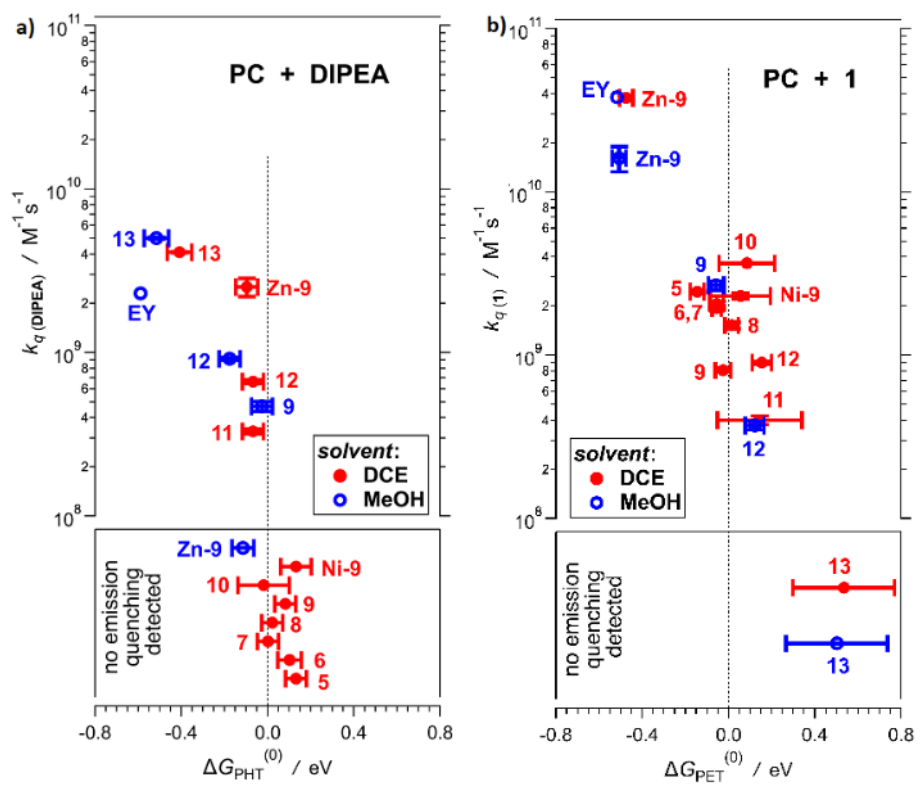

Figure 7. Correlation between the emission-quenching rate constants (obtained from the SV analyses) and the driving forces for the photoinduced charge transfer steps (estimated using eq. 1) for DCE and MeOH.

An increase in the solvent polarity causes a negative shift in the values of $\Delta G_{\mathrm{PHT}}{ }^{(0)}$ and $\Delta G_{\mathrm{PET}}{ }^{(0)}$ for each of the PCs. For example, $\Delta G_{\mathrm{PHT}}{ }^{(0)}$ for 9 is positive for DCE and negative for $\mathrm{MeOH}$ (Figure 7a), which agrees well with the SV analysis showing quenching with DIPEA for MeOH but not for DCE (Table 3). Because for PET $W=0$, we ascribe this trend to the improved electron donating properties of the photoexcited PCs with an increase in medium polarity. Indeed, a polarity increase makes the pyridinium ion a worse electron acceptor, as evident from the $30 \mathrm{mV}$ positive shift in its reduction potential when switching from DCM to MeOH. This magnitude of the observed shift, however, is way too small not only for the generalized Born radius $(\sim 0.21 \mathrm{~nm})$, but also for the hydrodynamic radius $(\sim 0.39 \mathrm{~nm})$, of the $N$-alkyl-2,4,6triphenylpyridinium acceptor (1), considering the changes in the Born solvation energy.[ref] We ascribe this lack of strong polarity dependence of the reduction potential to ion pairing that would become more pronounced with a decrease in medium polarity. ${ }^{37}$ That is, inducing ion pairing with a decrease in solvent polarity impedes the improvement of the electron-accepting propensity of pyridinium. Such ion pairing should be more prevalent for the pyridinium ions near or within the double layer at surface of the working electrode (for which the reduction 
potentials are representative) than for $\mathrm{Py}^{+}$in the bulk. Because the ion pairing makes pyridinium worse electron acceptor, the measured electrode potentials may be more negative than the reduction potentials representing the bulk $\mathrm{Py}^{+}$properties. Nevertheless, the polarity-induced changes in the reduction potentials of the oxidized PCs appears to principally contribute to the $\Delta G_{S}$ term in eq. $1 \mathrm{a}$.

In the case of PHT, on the other hand, an increase in solvent polarity: (1) makes $W$ less negative due to screening of the electrostatic interaction between the oxidized donor and reduced acceptor; and (2) shifts the reduction potentials making each ${ }^{1} \mathbf{P C} *$ a better electron acceptor and DIPEA a better electron donor. Our results reveal that for PHT between the porphyrins and DIPEA, the latter effect prevails.

The most electron-rich PC, Zn-9, however, presents a discrepancy in the observed PHT and PET trends. While an increase in the solvent polarity causes about $20 \mathrm{meV}$ negative shift in $\Delta G_{\mathrm{PHT}}{ }^{(0)}$ (concurring with what we observe for the other PCs), the SV analysis reveals that DIPEA quenches the Zn-9 emission in DCE but not in MeOH (Table 3). Changing from DCE to $\mathrm{MeOH}$ increase the reorganization energy by about $0.2 \mathrm{eV}$, which places the PHT kinetics for this solvent further from the tip of the Marcus curve. This trend, indeed, induces a decrease in the rates of PHT for $\mathrm{MeOH}$, but not to an extent where the quenching of the $\mathbf{Z n - 9}$ emission by DIPEA is not detectable at all, even for the relatively short excited-state lifetime that this PC has.

Examination of the bimolecular nature of the CT processes reveals a potential underlying reasons for the observed anomalous behavior of porphyrin Zn-9. The steady-state absorption spectra of complex Zn-9 for the two different solvents show ground-state aggregation in the presence of the salt 1 for DCE and $\mathrm{MeOH}$, and in the presence of DIPEA only for DCE (Figure 5). Conversely, the results do not exhibit any signs of aggregation of $\mathbf{Z n - 9}$ with DIPEA in $\mathrm{MeOH}$ (Figure 5). These findings reveal the importance of a static mechanism for complex $\mathbf{Z n -}$ 9 to undergo photoinduced CT with DIPEA. Dynamic collisional pathways most likely do not provide a long enough residence time of the ${ }^{1} \mathrm{PC}^{*}$ and DIPEA in proximity to each other or large enough donor-acceptor electronic coupling, to ensure efficient $\mathbf{C T}$.

The values of $\Delta G_{\mathrm{PET}}{ }^{(0)}$ and $\Delta G_{\mathrm{PHT}}{ }^{(0)}$, along with the SV analysis, reveal which pathway, PETHT or PHT-ET, prevails. Indeed, the electron-rich PCs would preferentially catalyze the reaction via PET-HT mechanism, while the electron-deficient ones - via PHT-ET (Figure 7). 
For operational PET-HT mechanism, however, $\Delta G_{\mathrm{PET}}{ }^{(0)}$ and $\Delta G_{\mathrm{HT}}^{(0)}$ should assume negative values. If $\Delta G_{\mathrm{PET}}^{(0)}<0$ and $\Delta G_{\mathrm{HT}}^{(0)}>0$, the $\mathrm{SV}$ analysis will still reveal emission quenching with the pyridinium salt $\mathbf{1}$, but the deaminative alkynylation would not proceed with detectable yields. Non-operational HT causes buildup of PET-generated $\mathbf{P C}^{\cdot+}$ and prevents the turnover of the catalyst. In a similar manner, $\Delta G_{\mathrm{PHT}}^{(0)}$ and $\Delta G_{\mathrm{ET}}{ }^{(0)}$ should assume negative values for operational PHT-ET mechanism, and $\Delta G_{\mathrm{PHT}}{ }^{(0)}<0$ and $\Delta G_{\mathrm{ET}}{ }^{(0)}>0$ will lead to buildup of PHTgenerated $\mathbf{P C}^{\bullet}$. Because for the porphyrin PCs $E_{00}<E_{\mathrm{ECH}}, \Delta G_{\mathrm{ET}}{ }^{(0)}<0$ if $\Delta G_{\mathrm{PET}}^{(0)} \lesssim 0$ and $\Delta G_{\mathrm{HT}}{ }^{(0)}<0$ if $\Delta G_{\mathrm{PHT}}{ }^{(0)} \lesssim 0$ (Figure 8). This feature ensures that for most PCs in this study the HT and ET steps will occur after the initial PET and PHT, respectively, and it holds for all PCs except 13. While 13 can catalyze the reaction and produce the yields we observe only via PHT-ET mechanism, it shows $\Delta G_{\mathrm{ET}}^{(0)}>0$ (Figure 8 ). The uncertainty in the estimates of the reduction potentials of the pyridinium salt $\mathbf{1}$ could be a source of this discrepancy. This findings illustrate the need for detailed understanding of what measured reduction potentials (of charged species, for example) represent, in order to ensure the predictive capabilities of the thermodynamic CT analysis (eq. 1). Because of the drastic differences between the timescales of the PCT processes and the rest of the reaction steps, the predictive power of the CT analysis is binary in nature. That is, if $\Delta G_{\mathrm{PCT}}{ }^{(0)}$ and $\Delta G_{\mathrm{CT}}{ }^{(0)}$ of sequential steps assume negative values, the reaction will occur; and if they do not - it will not. Even with miniscule PCT quantum yields, after hours of light radiation and a large enough number of turnover cycles, the buildup of photogenerated pyridinium radicals, A (Scheme 2), ensure the large product yields that we observe (Table 2). Indeed, the photostability of the PC and the chemical stability of its oxidized and reduced forms under the reaction conditions becomes a key requirement for achieving acceptably high product yields. Therefore, reversibility of electrochemical reduction or oxidation, along with a lack of photobleaching, serves as a first indication whether a photocatalyst may be acceptable, even if its electronic properties match perfectly with those of the substrates.

The outcomes of the thermodynamic CT analysis (Figure 7 and 8) illustrates the interplay between the two parallel pathways for light-driven shuttling of electrons from DIPEA to the pyridinium ions (Scheme 2). Switching between PET-HT and PHT-ET mechanisms reveals how PCs with different electronic properties drive the same photocatalytic reaction to completion with similar overall yields (Table 2). Furthermore, collectively the outcomes from the thermodynamic CT calculations (eq. 1), the SV studies (Table 3), and trends in the optical spectra (Figure 6) unveils mechanistic details, such as the importance of ground-state binding 
between the PC and the substrate, that each of these individual analyses cannot readily predict on its own.

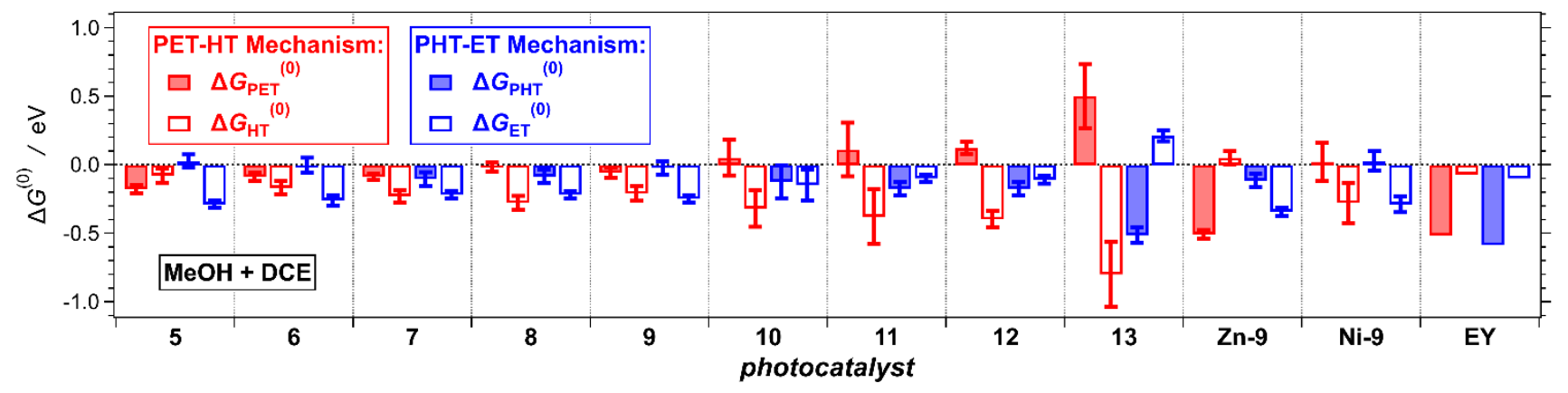

Figure 8. Driving forces for the CT steps involving the different photocatalysts as estimated using eq. 1 for $\mathrm{MeOH} / \mathrm{DCE}$ mixture, $\chi_{\mathrm{DCE}}=0.2$.

\section{CONCLUSIONS}

Photochemical reactions are extremely sensitive to the match between the electronic properties of the photocatalysts and the substrates. Charge transfer is the most important process for energy conversion, ensuring the functionality of light-harvesting system. Concurrently, to utilize the harvested energy, it is essential for the charge-transfer products to do chemical or other forms of work, such as formation of carbon-carbon bond from activated ammines and alkynes. ${ }^{26}$ Indeed, the importance of light-driven charge transfer extends considerably beyond energy conversion and storage. Via electronically excited states, photoinduced processes provide a broad access to products that are impossible to obtain by any other means. This work shows how the prevalent mechanism of a light-driven reaction adjusts to the changes in the electrochemical characteristics of the photocatalyst. This feature, involving parallel chargetransfer relays, significantly broadens the choices for photocatalysts, i.e., electron-rich and electron-deficient PCs drive the same reaction with similar efficiencies via different CT mechanisms (Scheme 2). Hence, the rational choice of a photoredox catalyst involves multifaceted analyses that extend considerably beyond the examination of its propensity to reduce or oxidize a substrate. These outcomes show the key importance of multifaceted mechanistic inquiries and set key paradigms not only for energy conversion and storage, but also for the development of synthetic methodologies.

\section{AUTHOR INFORMATION}

\section{Corresponding Author}


*D.G.: e-mail, dorota.gryko@icho.edu.pl; web, http://ww2. icho.edu.pl/gryko_group.

*V.I.V. e-mail: vullev@ucr.edu

\section{Funding Sources}

Foundation for Polish Sciences, National Science Center (PL), National Science Foundation (USA)

\section{Notes}

The authors declare no competing financial interest.

\section{ACKNOWLEDGMENT}

Financial support for this work was provided by the Foundation for Polish Sciences (FNP TEAM POIR.04.04.00-00-4232/17-00), the National Science Center (PL) KRJ Etiuda, UMO2017/24/T/ST5/00032, and the U.S.A. National Science Foundation (grants CHE 1800602 and CHE 1465284). V.I.V. thanks the Fulbright Commission Brazil, and the authors extend their gratitude to Dr. Maksymilian Karczewski and Dr. Eli M. Espinoza for computing the radii of the redox species used for this work.

\section{REFERENCES}

(1) Zhang, X.; Rakesh, K. P.; Ravindar, L.; Qin, H. L. Visible-light initiated aerobic oxidations: A critical review. Green Chem. 2018, 20 (21), 4790-4803.

(2) Michelin, C.; Hoffmann, N. Photocatalysis applied to organic synthesis - A green chemistry approach. Curr. Opin. Green Sustain. Chem. 2018, 10, 40-45.

(3) Reckenthäler, M.; Griesbeck, A. G. Photoredox catalysis for organic syntheses. Adv. Synth. Catal. 2013, 355 (14-15), 2727-2744.

(4) Ghosh, T.; Slanina, T.; König, B. Visible light photocatalytic reduction of aldehydes by Rh(III)-H: A detailed mechanistic study. Chem. Sci. 2015, 6 (3), 2027-2034.

(5) Xie, J.; Jin, H.; Hashmi, A. S. K. The recent achievements of redox-neutral radical C-C cross-coupling enabled by visible-light. Chem. Soc. Rev. 2017, 46 (17), 5193-5203.

(6) Wang, C. S.; Dixneuf, P. H.; Soulé, J. F. Photoredox Catalysis for Building C-C Bonds from C(sp2)-H Bonds. Chem. Rev. 2018, 118 (16), 7532-7585.

(7) Fagnoni, M.; Dondi, D.; Ravelli, D.; Albini, A. Photocatalysis for the Formation of the $\mathrm{C}-\mathrm{C}$ Bond Chem. Rev. 2007, 107 (6), 2725-2756.

(8) Zhu, J.; Yang, W. C.; Wang, X. D.; Wu, L. Photoredox Catalysis in C-S Bond Construction: Recent Progress in Photo-Catalyzed Formation of Sulfones and Sulfoxides. Adv. Synth. Catal. 2018, 360 (3), 386-400. 
(9) Zhao, Y.; Xia, W. Recent advances in radical-based C-N bond formation: Via photo/electrochemistry. Chem. Soc. Rev. 2018, 47 (8), 2591-2608.

(10) Revathi, L.; Ravindar, L.; Fang, W. Y.; Rakesh, K. P.; Qin, H. L. Visible Light-Induced C-H Bond Functionalization: A Critical Review. Adv. Synth. Catal. 2018, 360 (24), 4652 4698

(11) Qin, Q.; Jiang, H.; Hu, Z.; Ren, D.; Yu, S. Functionalization of C-H Bonds by Photoredox Catalysis. Chem. Rec. 2017, 17 (8), 754-774.

(12) König, B. Photocatalysis in Organic Synthesis - Past, Present, and Future. European J. Org. Chem. 2017, 15, 1979-1981.

(13) Ghosh, I.; Bardagi, J. I.; König, B. Reply to 'Photoredox Catalysis: The Need to Elucidate the Photochemical Mechanism'. Angew. Chemie Int. Ed. 2017, 56 (42), 12822-12824.

(14) Buzzetti, L.; Crisenza, G. E. M.; Melchiorre, P. Mechanistic Studies in Photocatalysis. Angew. Chem. Int. Ed. 2019, 58 (12), 3730-3747

(15) Arias-Rotondo, D. M.; McCusker, J. K. The photophysics of photoredox catalysis: A roadmap for catalyst design. Chem. Soc. Rev. 2016, 45 (21), 5803-5820.

(16) Nacsa, E. D.; MacMillan, D. W. C. Spin-Center Shift-Enabled Direct Enantioselective $\alpha-$ Benzylation of Aldehydes with Alcohols. J. Am. Chem. Soc. 2018, 140 (9), 3322-3330.

(17) Singh, A.; Teegardin, K.; Kelly, M.; Prasad, K. S.; Krishnan, S.; Weaver, J. D. Facile synthesis and complete characterization of homoleptic and heteroleptic cyclometalated Iridium(III) complexes for photocatalysis. J. Organomet. Chem. 2015, 776, 51-59.

(18) Gorbe, M.; Costero, A. M.; Sancenón, F.; Martínez-Máñez, R.; Ballesteros-Cillero, R.; Ochando, R. E.; Chulvi, K.; Gotor, R.; Gil, S. Halogen-containing BODIPY derivatives for photodynamic therapy Dye. Pigment. 2019, 160, 198-207.

(19) McCarthy, B. G.; Pearson, R. M.; Lim, C.-H.; Sartor, S. M.; Damrauer, N. H.; Garret, M. M. Structure-property relationships for tailoring phenoxazines as reducing photoredox catalysts. J. Am. Chem. Soc. 2018, 140 (15), 5088-5101.

(20) Joshi-Pangu, A.; Lévesque, F.; Roth, H. G.; Oliver, S. F.; Campeau, L. C.; Nicewicz, D.; DiRocco, D. A. Acridinium-Based Photocatalysts: A Sustainable Option in Photoredox Catalysis. J. Org. Chem. 2016, 81 (16), 7244-7249.

(21) Speckmeier, E.; Fischer, T. G.; Zeitler, K. A Toolbox Approach To Construct Broadly Applicable Metal-Free Catalysts for Photoredox Chemistry: Deliberate Tuning of Redox Potentials and Importance of Halogens in Donor-Acceptor Cyanoarenes. J. Am. Chem. Soc. 2018, 140 (45), 15353-15365.

(22) Osterloh, F. E. Photocatalysis versus Photosynthesis: A Sensitivity Analysis of Devices for Solar Energy Conversion and Chemical Transformations. ACS Energy Lett. 2017, 2 (2), 445-453.

(23) Rybicka-Jasińska, K.; Shan, W.; Zawada, K.; Kadish, K. M.; Gryko, D. Porphyrins as Photoredox Catalysts: Experimental and Theoretical Studies. J. Am. Chem. Soc. 2016, 138 (47), 15451-15458.

(24) Rybicka-Jasińska, K.; König, B.; Gryko, D. Porphyrin-Catalyzed Photochemical C-H Arylation of Heteroarenes. European J. Org. Chem. 2017, 2017 (15), 2104-2107.

(25) Clennan, E. L.; Pace, A. Advances in singlet oxygen chemistry. Tetrahedron 2005, 61 (28), 6665-6691.

(26) Ociepa, M.; Turkowska, J.; Gryko, D. Redox-Activated Amines in $\mathrm{C}\left(s p^{3}\right)-\mathrm{C}(s p)$ and $\mathrm{C}(s p$ $\left.{ }^{3}\right)-\mathrm{C}\left(s p^{2}\right)$ Bond Formation Enabled by Metal-Free Photoredox Catalysis. ACS Catal. 2018, 8 (12), 11362-11367

(27) Derr, J. B.; Tamayo, J.; Espinoza, E. M.; Clark, J. A.; Vullev, V. I. Dipole-induced effects on charge transfer and charge transport. Why do molecular electrets matter? Can. J. Chem. 2018, 96 (9), 843-858. 
(28) Alberty, R. A.; Hammes, G. G. Application of the theory of diffusion-controlled reactions to enzyme kinetics. J. Phys. Chem. 1958, 62 (2), 154-159.

(29) Majek, M.; Filace, F.; Von Wangelin, A. J. On the mechanism of photocatalytic reactions with eosin y. Beilstein J. Org. Chem. 2014, 10, 981-989.

(30) Volke, J.; Dunschb, L.; Heyrovsky, J.; Petrb, A.; Urbana, J. Comproportionation in the reduction of pyridinium combined ESR and electrochemical study. Electrochimica Acta, 1997, 42 (12), 1771-1780.

(31) Slyusareva, E. A.; Tomilin, F. N.; Sizykh, A. G.; Tankevich, E. Y.; Kuzubov, A. A.; Ovchinnikov, S. G. The effect of halogen substitution on the structure and electronic spectra of fluorone dyes. Opt. Spectrosc. 2012, 112 (5), 671-678.

(32) Espinoza, E. M.; Clark, J. A.; Soliman, J.; Derr, J. B.; Morales, M.; Vullev, V. I. Practical Aspects of Cyclic Voltammetry: How to Estimate Reduction Potentials When Irreversibility Prevails. J. Electrochem. Soc. 2019, 166 (5), H3175-H3187.

(33) Uno, B.; Okumura, N. Molecular Scientific Approach in Electroorganic Chemistry. Recent Res. Dev. Pure Appl. Chem. 2, 1998, 83-99.

(34) Espinoza, E. M.; Larsen, J. M.; Vullev, V. I. What Makes Oxidized N-Acylanthranilamides Stable? J. Phys. Chem. Lett. 2016, 7 (5), 758-764.

(35) Bao, D.; Millare, B.; Xia, W.; Steyer, B. G.; Gerasimenko, A. A.; Ferreira, A.; Contreras, A.; Vullev, V. I. Electrochemical oxidation of ferrocene: A strong dependence on the concentration of the supporting electrolyte for nonpolar solvents. J. Phys. Chem. A 2009, 113 (7), 1259-1267.

(36) Rehm, D.; Weller, A. Kinetics of fluorescence quenching by electron and H-atom transfer. Israel J. Chem. 1970, 8 (2), 259-271.

(37) Bird, M. J.; Iyoda, T.; Bonura, N.; Bakalis, J.; Ledbetter, A. J.; Miller, J. R. Effects of electrolytes on redox potentials through ion pairing. J. Electroanal. Chem. 2017, 804, 107-115. 